\title{
Pattern of Hematological Manifestations in Patients with Systemic Lupus Erythematosus Attending in a Tertiary Care Hospital
}

\author{
ABUL BASHAR MOHAMMAD ABU SUFIAN, ${ }^{1}$ M.A. KASHEM, ${ }^{2}$ SARMISTHA BISWAS ${ }^{3}$
}

\begin{abstract}
Background: Hematological manifestations are not uncommon with systemic lupus erythematosus (SLE). Some of them are life-threatening, warranting prompt action, while others need special consideration. This study aimed at pattern of hematological manifestations in patients with SLE.

Materials and Methods: This descriptive cross sectional study included patients who were diagnosed and treated as SLE at Dhaka Medical College Hospital. Detailed history and physical examinations were performed in every patient. SLE was diagnosed as per ACR criteria. Necessary investigations were carried out. Hematological manifestations were scrutinized and statistically analyzed.

Results: $A$ total of 89 patients ( $97.8 \%$ females, mean age $24.86 \pm 5.88$ years) were studied. Hematological manifestations were present in 77 (85.4\%) patients at the time of diagnosis. Among them anemia was most frequent in $77.5 \%$ patients followed by neutropenia (19.1\%), lymphopenia (17.9\%), thrombocytopenia (I7.9\%), autoimmune hemolytic anemia (AIHA) in $10.1 \%$ and pancytopenia in $5.6 \%$ patients. Normocytic normochromic anemia (NNA) was the most common peripheral blood film (PBF) findings found in 31 (34.8\%) of the patient, followed by microcytic hypochromic anemia (MHA) (32.6\%).

Conclusion: Hematological manifestations are frequently encountered in SLE. A sound conception about the relative frequency of hematological manifestations associated with systemic lupus erythematosus will help the clinician to take necessary measures for the wellbeing of the patient.
\end{abstract}

Key words: Systemic Lupus Erythematosus; hematological manifestations; anemia; leucopenia; thrombocytopenia.

\section{Introduction:}

Systemic lupus erythematosus (SLE) is a systemic autoimmune disease in which organs and cells undergo damage mediated by tissue-binding autoantibodies and immune complexes. Ninety percent of patients are women of child-bearing years; people of both sexes, all ages, and all ethnic groups are susceptible. ${ }^{1}$

The first classification criteria for SLE were developed by the American Rheumatism Association (predecessor of the American College of Rheumatology (ACR)) in 1971. ${ }^{2}$ Immunological tests were incorporated into the criteria and revised SLE classification criteria were published in $1982 .{ }^{3}$ The criteria, in 1997, underwent another revision and included advancing knowledge about the association of

1. Postgraduate Trainee, Dept. of Medicine, Dhaka Medical College \& Hospital.

2. Associate Professor, Dept. of Medicine, Dhaka Medical College \& Hospital.

3. Assistant Professor, Dept. of Medicine, Dhaka Medical College \& Hospital.

Corresponding author: Dr. Abul Bashar Mohammad Abu Sufian, Postgraduate Trainee, Dept. of Medicine, Dhaka Medical College \& Hospital. Cell: 01727238963, E-mail: abusufian6406@ gmail.com. antiphospholipid (aPL) antibodies with SLE. ${ }^{4}$ Although the criteria are widely accepted and used, only a few potential manifestations of SLE are represented. The Systemic Lupus International Collaborating Clinics (SLICC) SLE classification criteria comprise 11 clinical and 6 immunological criteria. ${ }^{5}$ In contrast to the ACR criteria, the SLICC criteria require at least one clinical and one immunological criteria for the classification of SLE. ${ }^{3,5}$ Autoimmune haemolytic anemia (AIHA), leucopenia and thrombocytopenia are part of both ACR and SLICC criteria.

Haematological abnormalities are common findings in patients with SLE. Sometimes, haematological abnormalities can be caused by the pathophysiology of SLE itself, but at other times they can be found in patients with SLE but not be a manifestation of SLE. According to the ACR and SLICC criteria for classification of SLE, leucopenia is defined as $<4000 / \mathrm{mm}^{4}$ on two or more occasions. Along with the pathogenic mechanism of disease itself, several other factors such as immunosuppressive drugs may contribute towards low white cell count in these patients. Leucopenia, that is, low total white blood cell (WBC) count, constitutes a paucity of granulocytes as well as lymphocytes, yet a greater absolute deficiency of granulocytes than lymphocytes is usually found. ${ }^{6}$ 
Granulocytopenia, or neutropenia is the most common WBC abnormality among patients with SLE. Neutropenia is usually defined as an absolute neutrophil count $<1000$ cells $/ \mathrm{cmm}$. Three potential mechanisms of neutropenia in SLE are (1) increased peripheral destruction of granulocytes; (2) changes in marginal and splenic pool, or increased margination; and (3) decreased marrow production. ${ }^{7}$

Neutropenia can be one of the contributing factors towards the infectious comorbidity in SLE. Recurrent infections are the only known significant consequence of neutropenia. Local signs and symptoms of infection-rubor, tumour, calor and dolor as originally described by Celsus in the firstcentury CE-may be attenuated in patients with SLE due to immunosuppression. Constitutional signs and symptoms of infection, such as fever, may also be absent in the immunocompromised patient. Hence, high vigilance is required.

Lymphopenia is defined as $<1.5 \times 10^{9}$ lymphocytes/L on two or more occasions according to the ACR and SLICC criteria. ${ }^{3,5}$ Low lymphocyte counts commonly occur in SLE with a prevalence ranging from $20 \%$ to $93 \%{ }^{8}$ and are observed frequently in patients with active or severe disease. ${ }^{9}$ Moreover, lymphocyte levels may fluctuate during the clinical course, irrespective of treatment. ${ }^{8}$ However, glucocorticoids and immunosuppressive drugs may contribute to the lymphopenia in severe disease. The degree of lymphopenia can be quite striking with values $<0.5 \times 10^{9}$ / L observed in $10 \%$ of patients. Lymphopenia occurs independently of neutropenia but may also contribute to the leucopenia seen in these patients. Neither set of criteria; however, specify how leucopenia and lymphopenia in these patients can be differentiated from decreased white cell count caused by immunosuppressive therapy or other causes. Thus, it is important to distinguish hematological abnormalities as either manifestations of SLE, consequence of SLE treatment or as part of another blood cell dyscrasia.

Presence of lymphopenia may be clinically silent or associated with increased risk of infections and/or active SLE. Data on the increased risk of infection are controversial and are complicated by the use of immunosuppressive therapies. Ethnicity may also play a role in explaining the conflicting results. Life-table analysis of patients in the Netherlands from 1991 showed no effect of lymphopenia on patient survival. ${ }^{10}$ In contrast, marked T cell depletion was associated with serious and often multiple infections in severely affected patients with SLE in India. ${ }^{9}$ However, high- dose glucocorticoids and cyclophosphamide use make these latter results difficult to interpret.

For the purpose of the ACR classification criteria for $\mathrm{SLE}^{3}$ and the new SLICC criteria, ${ }^{5}$ the definition of thrombocytopenia is a platelet count $<100 \times 10^{9} / \mathrm{L}$ without any other identifiable cause. Excluding thrombocytopenia as a result of pharmacological therapy may be especially difficult in patients with SLE. Pseudothrombocytopenia must be excluded by careful examination of the peripheral blood smear in order to determine whether platelet aggregation along with adherence to leucocytes has occurred.

True thrombocytopenia can occur by three mechanisms: impaired production of platelets in the bone marrow, sequestration of platelets in the spleen or accelerated destruction of platelets in the peripheral circulation. The majority of patients with SLE with thrombocytopenia have increased peripheral destruction that is commonly mediated by antiplatelet antibodies, but the other two mechanisms play a role in some patients. ${ }^{11}$

The ACR and SLICC criteria recognise AIHA with reticulocytosis as one of the criteria for the classification of SLE, while the SLICC criteria also include a positive Coombs test as a criterion. AIHA is one of the common aetiologies of severe anemia in patients with SLE. Reports regarding its diverse clinical presentation and heterogenous association to other autoimmune manifestations make prompt attention essential. Patients with AIHA present with constitutional signs and symptoms of anemia, including fatigue and dyspnoea on exertion. Patients with SLE with AIHA can have other concomitant autoimmune haematological manifestations. For example, patients with SLE can present with AIHA and thrombocytopenia concomitantly or sequentially, which is known as Evans syndrome. ${ }^{12}$ Patients with Evans syndrome may have frequent relapses, once glucocorticoids have been tapered or stopped. Hence, when a diagnosis of AIHA in patients with SLE has been established, monitoring for the development of thrombocytopenia is important.

\section{Materials and Methods:}

The study was carried out in 89 SLE patients. It was done from December 2015 to May 2016. All the samples were collected from the Medicine, Nephrology and Dermatology wards and SLE clinic of Dhaka Medical College Hospital. Patients of SLE as diagnosed according to American college of Rheumatology (ACR) criteria were included in the study and patients with hematological problems for due to other 
diseases were excluded. A structured questionnaire and necessary investigations were used as research instrument. Detailed history was collected in the structured questionnaire which includes demographic variables and co-morbidity. The form also included different physical signs found on examination ranging from general examination to systemic examinations. Data were presented by frequency and percentages and also diagram and charts. After compilation of data, the obtained data were checked, verified, edited and coded. The data were analyzed and statistical evaluation was performed by SPSS version 20.0 program.

\section{Result:}

Among the 89 enrolled patients 87 (97.8\%) were female and $2(2.2 \%)$ were male. Highest number of patients $(31.5 \%)$ was from the 21 to 25 years age group. Mean age $( \pm \mathrm{SD})$ was $24.86 \pm 5.88$ years (Table I). The most common presenting complaints were arthralgia $(80.9 \%)$ followed by malar rash (74.2\%) and myalgia (65.2\%) (Figure 1). Among the 89 patients, 51 patients were married at the time of diagnosis. Twelve $(23.5 \%)$ patients had a history of abortion. It has been found that of the diagnostic criteria, all the patients satisfied immunological criteria with anti-ds-DNA positivity. 98.8\% patients were positive for ANA followed by hematological abnormality (85.4\%), arthralgia (80.9\%), rash (74.2\%). Of 89 patients, 20 were hypertensive and 14 had autoimmune thyroiditis (Table II). A handful number of patients were taking disease modifying anti rheumatic drugs (DMARDs) and steroids that might play a role in causing cytopenia (Table III). Hematological abnormalities were detected in $76(85.4 \%)$ patients. The various abnormality included anemia, neutropenia, leucopenia (neutropenia and lymphopenia), thrombocytopenia, pancytopenia, Coombs test positivity and detection of antiphospholipid antibody. Anemia was the most common hematological abnormality detected in $69(77.5 \%)$ patients (Table IV). Autoimmune hemolytic anemia (AIHA) was diagnosed in 9 (10.1\%) patients. Sixty $(67.4 \%)$ patients had anemia due to various other causes including iron deficiency anemia (IDA) (32.6\%) and anemia of chronic disease (AOCD) (34.8\%). (Table V) Normocytic normochromic anemia (NNA) was the most common peripheral blood film (PBF) findings found in 31 (34.8\%) of the patients followed by microcytic hypochromic anemia (MHA) (32.6\%) (Table VI). Leukocyte count was found $>4000 / \mathrm{cmm}$ in $67(75.3 \%)$ patients while leucopenia was found in $21(23.6 \%)$ patients. (Table VII). A positive direct Coomb's test was found in $37 \%$ of the patients and antiphospholipid antibody (APLA) was positive in 19\% of the patients (Table VII).
Table I

Age distribution of patients $(n=89)$

\begin{tabular}{lccc}
\hline Age Category & Frequency & $\begin{array}{c}\text { Percentage } \\
(\%)\end{array}$ & $\begin{array}{c}\text { Cumulative } \\
\text { Percent }(\%)\end{array}$ \\
\hline $15-20$ & 23 & 25.8 & 25.8 \\
$21-25$ & 28 & 31.5 & 57.3 \\
$26-30$ & 17 & 19.1 & 76.4 \\
$31-35$ & 14 & 15.7 & 92.1 \\
$36-40$ & 7 & 7.9 & 100.0 \\
\hline Total & 89 & 100.0 & \\
\hline
\end{tabular}

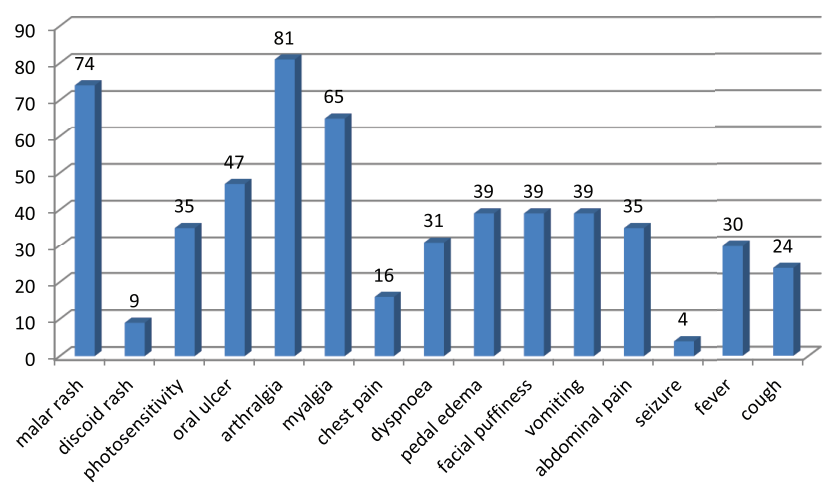

Fig.-1: Clinical features of the patients

Table II

Associated co-morbidities $(n=89)$

\begin{tabular}{lcc}
\hline Disease association & Frequency & Percent (\%) \\
\hline Hypertension & 20 & 22.5 \\
Autoimmune Thyroiditis & 14 & 15.7 \\
Diabetes Mellitus & 2 & 2.2 \\
Other & 16 & 18.0 \\
No history of any previous & 37 & 41.6 \\
disease/co-morbidity & & \\
\hline
\end{tabular}

Table III

Magnitude of the patient taking DMARDs $(n=89)$

\begin{tabular}{lcc}
\hline Name of DMARDS & Frequency & Percent (\%) \\
\hline Steroids & 74 & 83.1 \\
Hydroxychlroroquine & 28 & 31.5 \\
Cyclophosphamide & 22 & 24.7 \\
Methotrexate & 08 & 9.0 \\
Azathioprine & 04 & 4.0 \\
Mycophenolate Mofetil & 02 & 2.0 \\
\hline
\end{tabular}


Table IV

Pattern of hematological abnormalities $(n=76)$

\begin{tabular}{lcc}
\hline Hematological abnormality & Frequency & Percent (\%) \\
\hline Anemia & 69 & 77.5 \\
Leucopenia & 21 & 23.5 \\
Thrombocytopenia & 16 & 17.9 \\
Pancytopenia & 05 & 5.6 \\
\hline
\end{tabular}

Table V

Pattern of anemia $(n=69)$

\begin{tabular}{lcc}
\hline Pattern of anemia & Frequency & Percent (\%) \\
\hline Autoimmune hemolytic anemia & 9 & 10.1 \\
Iron deficiency anemia & 29 & 32.6 \\
Anemia of chronic disease & 31 & 34.8 \\
\hline
\end{tabular}

Table VI

Peripheral Blood film findings $(n=69)$

\begin{tabular}{lcc}
\hline Peripheral Blood film findings & Frequency & Percent (\%) \\
\hline Normocytic normochromic anemia & 31 & 34.8 \\
Microcytic hypochromic anemia & 29 & 32.6 \\
Macrocytic anemia & 09 & 10.1 \\
\hline
\end{tabular}

Table VII

Pattern of leukocyte abnormality $(n=89)$

\begin{tabular}{lcc}
\hline Leukocyte count & Frequency & Percent $(\%)$ \\
\hline Leukocyte count $(>4000 / \mathrm{cmm})$ & 67 & 75.3 \\
Leukocyte count $(<4000 / \mathrm{cmm})$ & 21 & 23.6 \\
Lymphopenia & 16 & 17.9 \\
Neutropenia & 17 & 19.1 \\
Both lymphopenia and neutropenia & 11 & 12.4 \\
\hline
\end{tabular}

Table VIII

Coomb's test and antiphospholipid antibody test $(n=89)$

\begin{tabular}{|c|c|c|}
\hline Name of the test & Frequency & $\begin{array}{c}\text { Percentage } \\
(\%)\end{array}$ \\
\hline
\end{tabular}

Coomb's test

- Positive

33

37.1

- Negative

56

62.9

Antiphospholipid antibody (IgG) test

- Positive 17

19.1

- Negative

72

80.9

\section{Discussion:}

This was a descriptive cross-sectional study conducted into inpatient department of Medicine, Nephrology, Dermatology and SLE clinic of Dhaka Medical College and Hospital to evaluate the hematological manifestations among 89 patients with SLE. There was a female preponderance in the studied patients, females were $97.8 \%$ and males $2.2 \%$. Male female ratio was 1:44. This is consistent with most of the studies. ${ }^{13}$ Cameron et al has reported a male to female ratio of $1: 8$ to 1: 14 in a series of adult patients. ${ }^{14}$

The mean $( \pm \mathrm{SD})$ age of the patients in this study was 24.86 $( \pm 5.88)$ years. . Highest number of patients $(31.5 \%)$ was in 21 to 25 years age group followed by 15 to 20 years of age (25.8\%). SLE is a disease of child bearing age. ${ }^{15}$ The median age of onset of SLE is 24 years in a series reported by Malaviya AN. ${ }^{16}$

The most common presenting complaints were arthralgia $(80.9 \%)$ followed by malar rash $(74.2 \%)$ and myalgia (65.2\%). Oral ulcer (47.8\%), facial puffiness (39.9\%), pedal edema (39.1\%), fever (30.5\%) were also common presenting symptoms. This is similar to some study done in India with a large sample size (321), where the presenting problems were the arthralgia (76\%) and then the fever $(61.9 \%) .{ }^{17} \mathrm{In}$ another study conducted at Chittagong Medical College Hospital by Md. Abdur Rauf et.al. ${ }^{12}$ the most common presenting complaints were photosensitivity $(84.2 \%)$ followed by fatigue $(50 \%)$ and rash $(45 \%)$. Fever $(44 \%)$ was also a common presenting symptoms with arthralgia $(41 \%){ }^{21}$

Oral ulcer was found in $47.8 \%$ in our patients consistent with Malviya et. al. ${ }^{16}$ In a series of studies done by Wallace ${ }^{18}$ oral ulcers were present in $7-36 \%$ of cases. ${ }^{18}$ Our study has documented a higher incidence of oral ulcers compared with western studies but the incidence is similar to that documented in the study from India. Malar rash was found in $74 \%$ of our patients. This observation is a bit higher to the series described by Wallace DJ where the incidence was $10-60 \%{ }^{18}$ Overall the relative frequency of each of the major clinical features at presentations was similar to what has been documented in literature, except from those which were conducted with small sample size.

Hypertension was the most common co-morbidity among the patients $(22.5 \%)$ followed by thyroid disorders $(21.3 \%)$. In analysis of nine published series of SLE patients, prevalence rate of hypertension $12-49 \%$ has been described. ${ }^{17}$ There is frequent association between hypertension and renal disease in SLE. In this study, out of 31 lupus nephritis patient, 20 patients were hypertensive. 
There were also 21 patients of thyroid disorder and 16 of them were having autoimmune thyroiditis. Two of our patients were also suffering from Diabetes Mellitus. An elaborate association could not be evaluated due to time and financial constraint.

The commonest criteria satisfied was anti ds-DNA (immunological) as evidenced by positivity in 100\% cases. 98.8\% patient showed ANA positivity; hence $1.2 \%$ were ANA negative lupus. This is not a very common expected finding as ANA negative lupus is a rare clinical entity. ANA positivity is reported to in about $90-95 \%$ of cases. ${ }^{17}$ Next to follow are the hematological abnormality (85.4\%), arthralgia $(80.9 \%)$, rash $(74.2 \%)$, oral ulcer $(47.8 \%)$, renal involvement $(30.9 \%)$.

Hematological abnormalities were detected in $85.4 \%$ patients diagnosed to have SLE in this study consistent with Xu XM et. al. ${ }^{19}$ Aleem et. al. studied 624 SLE patients for hematological abnormalities of which hematological abnormalities were present in $516(82.7 \%)$ patients at the time of diagnosis. ${ }^{20}$ Though there was such a high incidence of hematological features at presentation, only 4 patients presented primarily with symptom attributed to hematological abnormalities specially purpura.

Most common hematological abnormality was anemia (77.5\%). Various studies have shown a similar findings ${ }^{21}$. In a series of studies reviewed by BudMan anemia occurred in $57-78 \%$ of patients with $\mathrm{SLE}^{18}$. Aleem et. al. ${ }^{20}$ concluded; of hematological abnormalities, anemia was the most common disorder present in $63 \%$ of patients followed by lymphopenia in $40.3 \%$, leukopenia in $30.0 \%$, thrombocytopenia in $10.9 \%$ and autoimmune haemolytic anemia (AIHA) in $4.6 \%$ patients. $^{20}$

Iron deficiency anemia (IDA), anemia of chronic disease (AOCD) and autoimmune hemolytic anemia (AIHA) are the common cause of anemia in SLE. ${ }^{20}$ Iron deficiency has been diagnosed on the basis of hypochromicity on the peripheral smear mainly, along with some clinical features like pica, nail changes, glossitis and chelitis. Anemia of chronic disease on the basis of a normocytic and normochromic blood picture in the presence of negative Coomb's test and AIHA on the basis of positive Coomb's test and reticulocytosis. Further investigations to evaluate anemia such as iron studies, serum folate levels and bone marrow examinations has not been done due to financial constraints.

Anemia of chronic disease was the most common cause of anemia and accounted for $34.8 \%$ and iron deficiency anemia was $32.6 \%$. The finding is similar to that documented by Voulgarelis et al. ${ }^{22}$
A positive Coomb's test was observed in $37.1 \%$ of our patients but AIHA was documented in only $10.1 \%$. Budman $\mathrm{R}$ in a review of a series of studies has found 18 to $65 \%$ of Coomb's positivity in patients with SLE. However the incidence of AIHA in his study was less than $10 \% .{ }^{21}$ Aleem et. al. ${ }^{20}$ found a positive Coombs' test was found in 80 (35.9\%) of the 223 patients tested, and 29/624 (4.6\%) patients developed autoimmune hemolytic anemia (AIHA). ${ }^{20}$ Three of our patients developed Evans syndrome (3\%) which was higher than that of Aleem et. al. $[3(0.5 \%)] .{ }^{20}$

Reticulocytosis was one of the diagnostic criteria for the diagnosis of AIHA. The bone marrow response to hemolysis in the form of reticulocytosis may be depressed if associated or folate deficiency is present or associated bone marrow suppression of chronic disease is present. This could be responsible for the gross discrepancy seen between the incidence of Coomb's positivity and the incidence of AIHA. More sophisticated test would perhaps have documented hemolysis in a greater number of patients.

Neutropenia was predominant $(51.5 \%)$ among the WBC disorder. In a review by Katsanis et al it has been conclude that in SLE greater absolute deficiency of granulocytes than lymphocytes is usually found. ${ }^{6}$ But in another study by Debarre et. al. lymphopenia was detected in $84 \%$ of the cases and was found to be independent of leucopenia. ${ }^{23}$ Thrombocytopenia was detected in $18 \%$ of patients but 4 of them had severe thrombocytopenia $(<20,000 / \mathrm{cmm})$ with purpuric spot at presentation. Budman $\mathrm{R}$ describes an incidence of $14-26 \%$ thrombocytopenia in different series. ${ }^{21}$ The causes of thrombocytopenia and white cell abnormalities are usually immune in nature and result from destruction of antibody coated cells. ${ }^{24}$ Thrombocytopenia in SLE may also be due to drugs, infection and bone marrow suppression, and may be closely associated with hemolytic anemia or a manifestation of APS. ${ }^{25}$

$5.6 \%$ of our patients were having pancytopenia. A handful number of patients were taking disease modifying anti rheumatic agents (DMARDs) and steroids that might play a role in causing cytopenia. $83.1 \%$ of patients were taking steroids and $31.5 \% \quad \& \quad 24.7 \%$ were taking hydroxychloroquine \& cyclophosphamide respectively. Aleem et. al. ${ }^{20}$ found that most common causes of cytopenias during the course of SLE include drugs, infections and immune mediated. ${ }^{20}$ Drugs like cyclophosphamide and azathioprine were considered to be the cause of cytopenias in a substantial number of cases in this study.

Antiphospholipid antibodies (IgG) were positive in 19.1\% patients. Levin et al detected Antiphospholipid antibodies in $12-30 \%$ of cases in a study which is similar to our study. ${ }^{26}$ 


\section{Conclusion:}

Haematological abnormalities are common findings in patients with SLE. It is important to distinguish haematological abnormalities as either manifestation of SLE, consequence of SLE treatment or as a part of another blood dyscrasia. Anemia is common among the haematological manifestations of SLE patients. Leucopenia and thrombocytopenia are also evident. Similar surveys should be conducted to delineate the actual intensity of the problem with modern lab facilities, bigger sample size, with elimination of confounding variables. Moreover cohort studies should be undertaken to find natural history of the hematological manifestations and to evaluate the effect of therapeutic interventions upon it.

\section{Conflict of interest: None.}

\section{References:}

1. Kasper DL, Braunwald E, Fauci AS, Hauser SL, Longo DL, Jameson JL et.al. Systemic lupus erythematosus. In: Harrison's principles of internal medicine, 17th ed. New York: McGraw-Hill Medical Publishing Division. $2008 ; 2075$

2. Lie TH, Rothfield NF. An evaluation of the preliminary criteria for the diagnosis of systemic lupus erythematosus. Arthritis Rheum 1972;15:532-4.

3. Tan EM, Cohen AS, Fries JF. The 1982 revised criteria for the classification of systemic lupus erythematosus. Arthritis Rheum 1982;25:1271-7.

4. Feletar M, Ibanez D, Urowitz MB. The impact of the 1997 update of the American College of Rheumatology revised criteria for the classification of systemic lupus erythematosus: what has been changed? Arthritis Rheum 2003;48:2067-9.

5. Petri M, Orbai AM, Alarcon GS. Derivation and validation of the Systemic Lupus International Collaborating Clinics classification criteria for systemic lupus erythematosus. Arthritis Rheum 2012;64:2677-86.

6. Katsanis E, Hsu E, Luke KH. Systemic lupus erythematosus and sickle hemoglobinopathies: a report of two cases and review of the literature. Am J Hematol 1987;25:211-14.

7. Starkebaum G, Price TH, Lee MY. Autoimmune neutropenia in systemic lupus erythematosus. Arthritis Rheum 1978;21:504-12.

8. Rivero SJ, Diaz-Jouanen E, Alarcon-Segovia D. Lymphopenia in systemic lupus erythematosus. Clinical, diagnostic, and prognostic significance. Arthritis Rheum 1978;21:295-305.

9. Bhuyan UN, Malaviya AN. Deficiency of T cells in blood and tissues and severity of infections in systemic lupus erythematosus. Indian J Med Res 1978;67:269-78.

10. Nossent JC, Swaak AJ. Prevalence and significance of haematological abnormalities in patients with systemic lupus erythematosus. Q J Med 1991;80:605-12.
11. Wallace DJ, Hahn BH, Kapouzas GA. Hematological and Lymphoid Abnomalities in SLE. In: DUBOIS' Lupus erythmatosus and Related Syndomes. 8th edn. Philadelphia: Elsevier, 2013:426-37.

12. Rouf MA, Uddin AAMR, Hoque E, Ismail M, Biswas RSR, Alam $\mathrm{F}$ et. al. Pattern of hematological manifestations in patients with systemic lupus erythematosus attending in a tertiary care hospital. Chattagram Maa-O-Shishu Hospital Medical College Journal 2014;13:49-53.

13. Manzi M, Ramsay- Goldman, Star KV, Epidemiology of systemic lupus erythematosus. In Rheumatology, philadaelphia: Mosby. 2003;3:1295-96

14. Cameron JS. Disease of the Month: Lupus Nephritis. J.Am Soc Nephrol 1999; 10: 413-24.

15. Janz TG, Johnson RL, Rubenstein SD. "Anemia in the emergency department: evaluation and treatment.” Emerge med pract 2013;15 (11):1-15.

16. Malaviya AN, Singh RR, Kumar A. Systemic lupus erythematosus in Northern India. A review of 329 cases. J Assoc Physicians India.1988;36:473-475.

17. Kumar A. Indian guideline on the management of SLE. Indian Rheumatologicalm Assoc 2002;10:80-96.

18. Wallace DJ, The clinical presentations of SLE, In Dubois' Lupus erythematosus, Wallace DJ and Hahn BH (eds) Philadelphia, Lea and Febiger 1993;4(33):317-21.

19. Hu XM, Fan ZR, Zhou SY, Wei W, Zhu BH, Cao YF. Hematological abnormality and clinical characteristics in systemic lupus erythematosus. Zhongguo shi Yan. 2004;12 (2):170-73.

20. Aleem A, Al Arfaj AS, Khalil N, Alarfaj H. Haematological abnormalities in systemic lupus erythematosus. Acta Reumatol Port. 2014;39:236-241.

21. Budman DR and Steinberg AD. Hematologic aspects of systemic lupus erythematosus. Current concepts. Ann Int Med 1977;86:220-29.

22. Voulgarelis M, Kokori SIG, Ioannidis JPA. Anemia in systemic lupus erythematosus: etiological profile and the role of erytropoetin . Ann Rheum Dis 2000;59:217-22.

23. Miguel EC, Pereira RM, Pereira CA. Psychiatric manfestations of systemic lupus erythematosus: Clinical features, symptoms and signs of central nervous system activity in 43 patients. Medicine1994;73:224-32.

24 Liu H, Ozaki K, Matsuzaki Y, Abe M, Kosaka M, Saito S. Suppression of haematopoiesis by IgG autoantibodies from patients with systemic lupus erythematosus (SLE). Clin Exp Immunol 1995;100:480-85.

25. Pujol M, Ribera A, Vilardell M. High prevalence of platelet autoantibodies in patients with systemic lupus erythematosus. Br J Haematol 1995;89:137-41.

26. Levin JS, Fritzler, Elkon K. Autoantibodies in SLE, In Rheumatology, M.C. Hochberg, AJ Silman (eds) Philadelphia: Mosby 2003;3:1337-46. 